\title{
THE LITHIUM ISOTOPE RATIO IN OLD STARS
}

\author{
P.E. NISSEN \\ Institute of Physics and Astronomy, University of Aarhus, \\ DK-8000 Aarhus C, Denmark
}

The lithium isotope ratio in stars can be determined from high resolution observations of the profile of the $\mathrm{Li}$ I $6707 \AA$ absorption line. Earlier studies of old F and G stars (Andersen et al. 1984, Maurice et al. 1984, Pilachowski et al. 1989) have led to upper limits of ${ }^{6} \mathrm{Li} /{ }^{7} \mathrm{Li}$ ranging from 0.05 to 0.10 . Recently, Smith, Lambert \& Nissen (1993) seem to have detected ${ }^{6} \mathrm{Li}$ in HD 84937 - a metal-poor turnoff star with $T_{\text {eff }} \simeq 6200 \mathrm{~K}$ and $[\mathrm{Fe} / \mathrm{H}] \simeq-2.4$. An isotope ratio ${ }^{6} \mathrm{Li} /{ }^{7} \mathrm{Li}=0.05 \pm 0.02$ was determined (see Fig. 1). The detection has been confirmed by Hobbs \& Thorburn (1994), who derived ${ }^{6} \mathrm{Li} /{ }^{7} \mathrm{Li}=0.07 \pm 0.03$. The main contribution to the quoted $(1 \sigma)$ errors comes from the noise in the spectrum $(\mathrm{S} / \mathrm{N}=400)$ and possible errors in the Doppler broadening of the Li line (Nissen 1994). This broadening is due to stellar rotation and macro-turbulent motions in the stellar atmosphere and can be determined from the profiles of unblended metallic absorption lines.

As discussed in detail by Steigman et al. (1993) the presence of ${ }^{6} \mathrm{Li}$ in the atmosphere of HD 84937 is consistent with the measured Be abundance (Boesgaard \& King 1993) within the context of i) Standard Big Bang nucleosynthesis, ii) Pop. II cosmic ray nucleosynthesis and iii) standard (non-rotating) models for $\mathrm{Li}$ depletion. In particular, Steigman et al. derive $D_{6}>0.2$, where $D_{6}$ is the depletion factor for ${ }^{6} \mathrm{Li}$. As shown by Chaboyer (1994) standard stellar evolution models with new opacities predict $D_{6} \simeq 0.4$ for turnoff stars and subgiants with $T_{\text {eff }}>5900 \mathrm{~K}$. The same models predict $D_{7} \simeq 1.0$, i.e. no ${ }^{7} \mathrm{Li}$ depletion for main sequence stars as well as subgiants with $T_{\text {eff }} \geq 5800 \mathrm{~K}$.

Non-standard models with rotational induced mixing predicting a strong degree of ${ }^{7} \mathrm{Li}$ depletion $\left(D_{7} \simeq 0.1\right.$ ) (Pinsonneault et al. 1992) seem to be excluded by the detection of ${ }^{6} \mathrm{Li}$ in HD 84937 , because the same models predict a very severe ${ }^{6} \mathrm{Li}$ depletion $\left(D_{6}<0.01\right)$. Hence, inhomogeneous Big Bang models predicting $\log \varepsilon(\mathrm{Li}) \simeq 3.0$ can probably be excluded. 


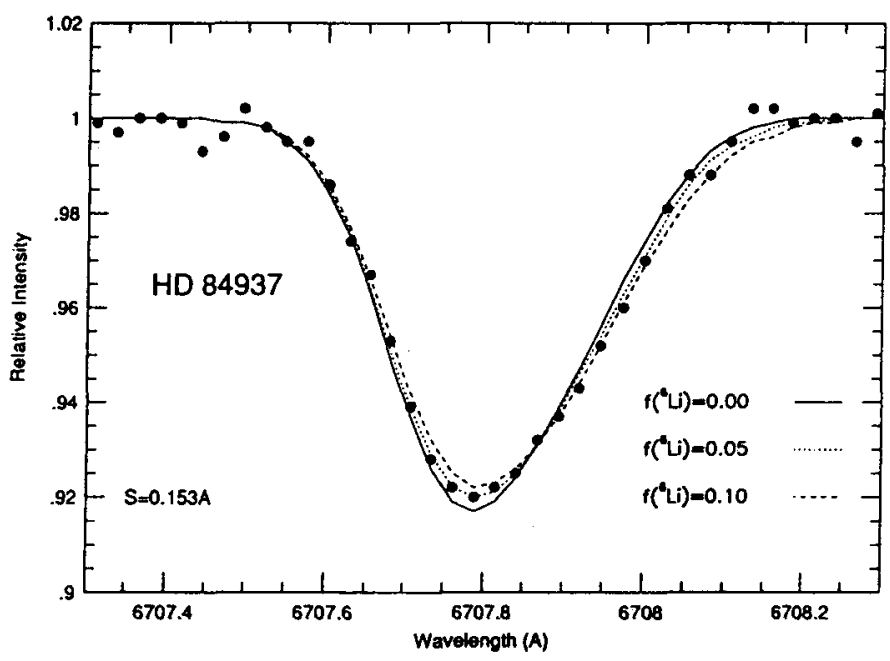

Figure 1. The observed profile ( $\bullet$ ) of the Li I line of HD 84937 compared with synthetic model atmosphere profiles for three ${ }^{6} \mathrm{Li} /{ }^{7} \mathrm{Li}$ ratios. $\mathrm{S}$ is the $\mathrm{FWHM}$ of the Gaussian smoothing function as determined from the observed profile of the $\mathrm{CaI} 6162 \AA$ line. See Smith, Lambert \& Nissen (1993) for details.

A few other metal-poor stars were included in the works of Smith et al. (1993) and Hobbs \& Thorburn (1994). In particular, one should note the upper limits ${ }^{6} \mathrm{Li} /{ }^{7} \mathrm{Li} \leq 0.02$ for $\mathrm{HD} 19445$ - a dwarf star with $T_{\text {eff }} \simeq 5800 \mathrm{~K}$, and ${ }^{6} \mathrm{Li} /{ }^{7} \mathrm{Li} \leq 0.03$ for HD 140283 - a subgiant with $T_{\text {eff }} \simeq 5700 \mathrm{~K}$. Both results are consistent with the measured Be abundances and standard models for stellar depletion of ${ }^{6} \mathrm{Li}$. Further studies of the $\mathrm{Li}$ isotope ratio in Pop. II stars are, however, needed to confirm that turnoff stars and the hottest subgiants do indeed have a small amount of ${ }^{6} \mathrm{Li}$ in their atmospheres. For this purpose very large telescopes equipped with high resolution $(R>100000)$ spectrometers are needed.

\section{References}

Andersen, J., Gustafsson, B., Lambert, D.L. 1984, A\&A 136, 65

Boesgaard, A.M., King, J.R. 1993, AJ 106, 2309

Chaboyer, B.C. 1994, ApJ Letters (submitted)

Hobbs, L.M., Thorburn, J.A. 1994, ApJ 428, L25

Maurice, E., Spite, F., Spite, M. 1984, A\&A 132, 278

Nissen, P.E. 1994, in "The Light Element Abundances", ed. P. Crane, ESO Conf. Proc., Lecture Notes in Physics, Springer-Verlag (in press)

Pilachowski, C.A., Hobbs, L.M., De Young, D.S. 1989, ApJ 345, L39

Pinsonneault, M.H., Deliyannis, C.P., Demarque, P. 1992, ApJS 78, 179

Smith, V.V., Lambert, D.L., Nissen, P.E. 1993, ApJ 408, 262

Steigman, G., Fields, B.D., Olive, K.A., Schramm, D.N, Walker, T.P. 1993, ApJ 415, L35 